\title{
CARACTERIZAÇÃO DO COMPORTAMENTO DE OXIDAÇÃO DE UMA LIGA INOXIDÁVEL Fe-Mn-Si-Cr-Ni-Co COM EFEITO DE MEMÓRIA DE FORMA EM ELEVADA TEMPERATURA
}

\section{CHARACTERIZATION ON OXIDATION BEHAVIOR OF A STAINLESS Fe-Mn-Si- Cr-Ni-Co SHAPE MEMORY ALLOY AT HIGH TEMPERATURE}

\author{
C. ARANA ${ }^{1}$, R. SILVA², C. A. DELLA ROVERE ${ }^{3}$ \\ ${ }^{1}$ Universidade Federal de São Carlos, Departamento de Engenharia de Materiais, Brasil \\ ${ }^{2}$ Universidade Federal de São Carlos, Departamento de Engenharia de Materiais, Brasil \\ ${ }^{3}$ Universidade Federal de São Carlos, Departamento de Engenharia de Materiais, Brasil \\ E-mail: arana.camila@hotmail.com
}

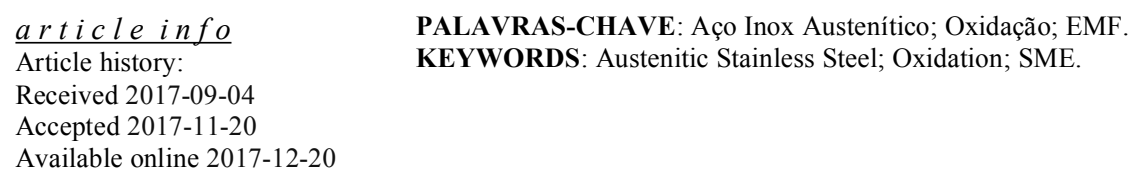

RESUMO: Os aços inoxidáveis austeníticos com alto teor de Mn e baixo teor de Ni têm atraído muita atenção recentemente. Estas ligas, além de possuírem boas propriedades mecânicas e resistência à corrosão, possuem memória de forma. No entanto, o desenvolvimento destas ligas ainda é relativamente recente, e muitas de suas propriedades precisam ser estudadas. Deste modo, o objetivo deste trabalho foi caracterizar o comportamento de oxidação de uma liga $\mathrm{Fe}-\mathrm{Mn}$-Si-Cr-Ni-Co com memória de forma em $800{ }^{\circ} \mathrm{C}$ ao ar. Os resultados obtidos mostram que o comportamento de oxidação do material estudado obedece a uma lei parabólica. Após $120 \mathrm{~h}$ de oxidação, a camada de óxido formada na superficie da liga é composta principalmente de óxidos de Mn. A oxidação da liga provoca a transformação de fase de austenita para ferrita em regiões empobrecidas em Mn.

\begin{abstract}
Austenitic stainless steels with high Mn content and low Ni content have been attracting much attention recently. These alloys, besides having good mechanical properties and corrosion resistance, also present shape memory effect. However, the development of alloys of this kind is still very recent, and further studies on its properties are needed. Thus, the aim of this work was to characterize the oxidation behavior of a $\mathrm{Fe}-\mathrm{Mn}$-Si-Cr-Ni-Co shape memory alloy at $800{ }^{\circ} \mathrm{C}$ in air. The obtained results showed that the oxidation behavior of the studied material obeys a parabolic law. After $120 \mathrm{~h}$ of oxidation, the oxide layer formed on the surface of the alloy is composed mainly by Mn oxides. The oxidation of the alloy causes phase transformation of austenite to ferrite in $\mathrm{Mn}$-depleted regions.
\end{abstract}

\section{INTRODUÇÃO}

Os aços inoxidáveis austeníticos são materiais estruturais de engenharia extremamente importantes, pois combinam boas propriedades mecânicas, soldabilidade e excelente resistência à corrosão e ao calor. Estes aços são amplamente utilizados na produção de 
energia e em indústrias de processamento químico onde são expostos a temperaturas elevadas na faixa 600 a $900{ }^{\circ} \mathrm{C}$. No entanto, esses materiais possuem custos de produção relativamente elevados, pois contém elevados teores de Ni, que é um elemento de alto custo e tem seu preço aumentado muito nos últimos anos (ZHANG et al., 2009; SILVA et al., 2016; MA et al., 2013). Este fato limita consideravelmente a aplicação destas ligas.

Nesse sentido, aços inoxidáveis austeníticos com alto teor de Mn e baixo teor Ni têm atraído muita atenção recentemente (MA et al., 2013). As ligas austeníticas inoxidáveis FeMn-Si-Cr-Ni com efeito de memória de forma (EMF) contendo elevado teor de Mn (13-20\% em peso) e teores relativamente baixos de $\mathrm{Ni}(<5 \%$ em peso) quando comparado com o convencional aço inoxidável austenítico, têm se tornado materiais promissores para diferentes aplicações industriais. Devido à combinação de propriedades únicas, tais como EMF, boas propriedades mecânicas, resistência à corrosão e baixo custo de produção (MA et al., 2013; ROVERE et al., 2012; ROVERE et al., 2011), esses materiais se tornaram potenciais candidatos para várias aplicações de engenharia, como por exemplo, em dispositivos para liberação de painéis solares em satélite e acoplamento de tubos sem solda.

No entanto, o desenvolvimento destas ligas ainda é relativamente recente, muitas das suas propriedades são razões de investigação, particularmente a resistência à oxidação em ambientes altamente oxidantes, como os encontrados em altas temperaturas nas plantas de produção de energia e processamento químico. Em vista disso, e tendo em conta a escassez de estudos sobre comportamento de oxidação em alta temperatura de ligas austeníticas $\mathrm{Fe}$ Mn-Si-Cr-Ni com EMF, este trabalho tem como objetivo caracterizar o comportamento de oxidação a $800{ }^{\circ} \mathrm{C}$ ao ar por até 120 h de uma liga austenítica Fe-8Mn-5Si-13Cr-5Ni-12Co com EMF.

\section{MATERIAL E MÉTODOS}

\subsection{Preparação das Amostras}

A liga estudada foi preparada por fusão em um forno de indução a vácuo (VIM), utilizando matéria prima de alta pureza. Seguido de forjamento a quente em $1200{ }^{\circ} \mathrm{C} \mathrm{em}$ forma de barra, e solubilização em $1050{ }^{\circ} \mathrm{C}$ por $1 \mathrm{~h}$ com resfriamento em água em temperatura ambiente. A Tabela 1 mostra a composição química do material estudado.

Tabela 1 - Composição Química da Liga (\% em peso).

\begin{tabular}{ccccccc}
\hline $\mathrm{Fe}$ & $\mathrm{Mn}$ & $\mathrm{Si}$ & $\mathrm{Cr}$ & $\mathrm{Ni}$ & $\mathrm{Co}$ & $\mathrm{C}$ \\
\hline Bal. & 8,26 & 5,25 & 12,8 & 5,81 & 11,84 & 0,009 \\
\hline
\end{tabular}


Para os testes de oxidação, foram utilizadas amostras cilíndricas de $6 \mathrm{~mm}$ de diâmetro e $10 \mathrm{~mm}$ de altura, obtidas através de usinagem por eletroerosão. Antes dos testes, as amostras foram lixadas mecanicamente nas granulometrias 400, 600 e 1200. Em seguida, foram desengorduradas com acetona. Os ensaios de oxidação foram realizados ao ar estático a $800^{\circ} \mathrm{C}$ por um período de exposiç̧ão de $5,10,24,48,72,96$ e $120 \mathrm{~h}$.

Antes da realização dos ensaios, as amostras foram colocadas individualmente em cadinhos de alumina fundidos para evitar perdas de massa geradas por possíveis trincas dos óxidos formados. Após os vários períodos de oxidação, os cadinhos, juntamente com as amostras, foram retirados do forno e resfriados ao ar até temperatura ambiente. As variações de massa resultantes da formação de óxido foram medidas em balança eletrônica com precisão de $0,1 \mathrm{mg}$. Os cálculos de ganho de massa médio e desvio padrão foram realizados com base em dois testes de oxidação para cada condição.

\subsection{Caracterização do Material}

Para a caracterização microestrutural, as amostras solubilizada e oxidadas por até $120 \mathrm{~h}$ foram embutidas em resina de poliéster de cura a frio, lixadas mecanicamente nas granulometrias 400, 600 e 1200, polidas com uma suspensão de alumina de $1 \mu \mathrm{m}$ e atacadas com reagente de Vilella $\left(5 \mathrm{~mL} 37 \% \mathrm{HCl}+95 \mathrm{~mL}\right.$ etanol $\left.+1 \mathrm{~g} \mathrm{C}_{6} \mathrm{H}_{3} \mathrm{~N}_{3} \mathrm{O}_{7}\right)$. As microestruturas resultantes foram examinadas em microscópio óptico (MO) e microscópio eletrônico de varredura (MEV). Adicionalmente, análises de difração de raios-X (DRX) foram realizadas na microestrutura da amostra solubilizada e sobre a camada de óxido formada na superfície da amostra oxidada a $800{ }^{\circ} \mathrm{C}$ por $120 \mathrm{~h}$. Os difratogramas foram

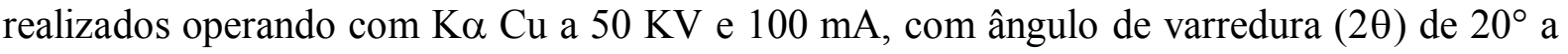
$100^{\circ}$ e velocidade de varredura de $2 \%$ min.

\section{RESULTADOS E DISCUSSÃO}

A Figura 1 mostra imagens de MO (a) e MEV (b) da amostra solubilizada do material estudado. Pelas imagens é possível observar que a microestrutura é composta essencialmente de austenita, onde é possível notar a presença de algumas maclas, e blocos de martensita- $\varepsilon$. A Figura 2 apresenta o padrão de DRX da amostra solubilizada, no qual é evidenciado a presença das fases austenita e martensita- $\varepsilon$. Microestruturas similares foram também reportadas por KAJIWARA et al., 1999 e MAJI et al., 2006 trabalhando com ligas Fe-Mn-SiCr-Ni com EMF. 


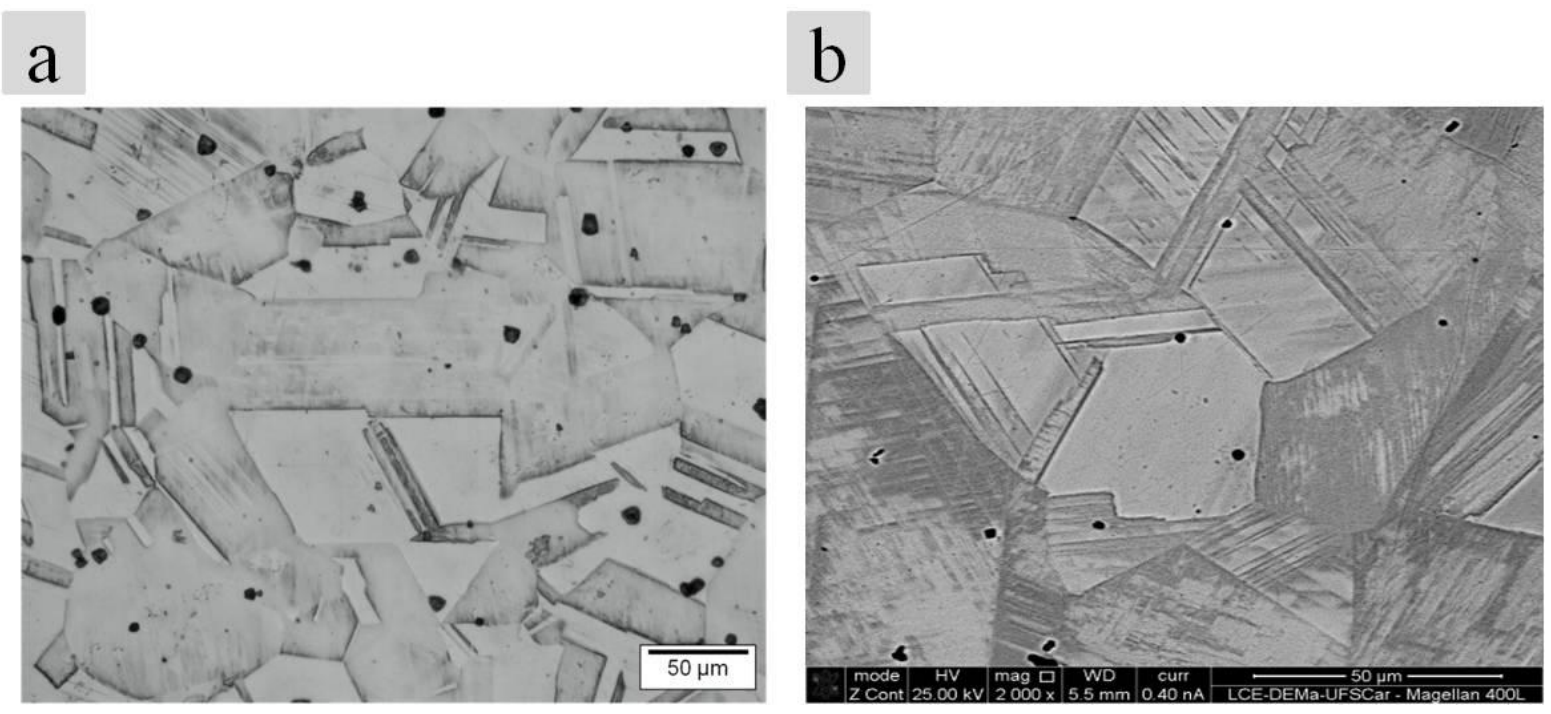

Figura 1 - Imagens de MO (a) e MEV (b) da amostra solubilizada.

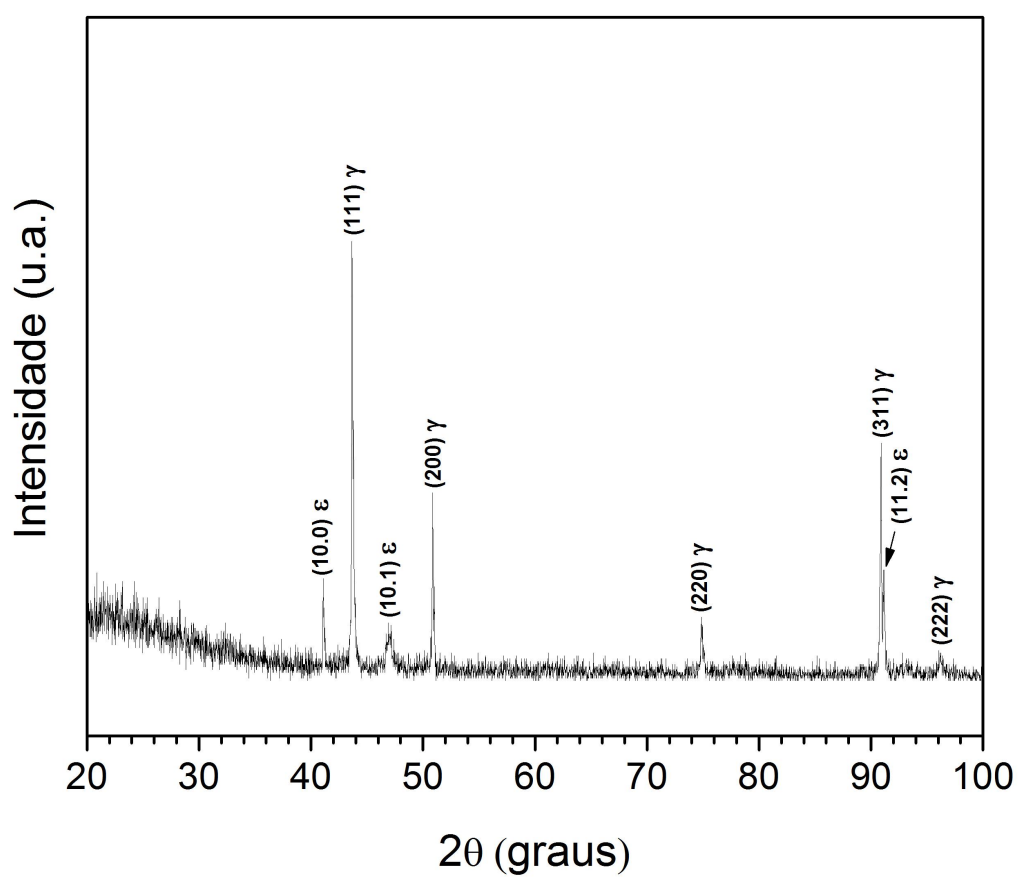

Figura 2 - Padrão de DRX da microestrutura da amostra solubilizada.

A Figura 3 (a) mostra o ganho da massa da liga Fe-Mn-Si-Cr-Ni-Co em função do tempo de oxidação a $800{ }^{\circ} \mathrm{C}$ ao ar. Note que o ganho de massa após oxidação por $120 \mathrm{~h}$ é de aproximadamente $0,82 \mathrm{mg} / \mathrm{cm}^{2}$. Este resultado mostra que a liga estudada possui baixa resistência à oxidação em $800{ }^{\circ} \mathrm{C}$ quando comparada com os dados reportados na literatura para o comumente utilizado 304 oxidado na mesma temperatura por até $100 \mathrm{~h}$ (cerca de 0,23 
$\mathrm{mg} / \mathrm{cm}^{2}$ ) (ISSARTEL et al., 2004). Entretanto, deve ser mencionado que o aço 304 possui elevado teor de $\mathrm{Cr}$ e baixo teor de $\mathrm{Mn}$ que são elementos que influenciam significativamente na resistência à oxidação em elevada temperatura (STOTT, et al., 1989). A Figura 3 (b) mostra o quadrado do ganho de massa da liga estudada, onde é possível observar um incremento linear com o tempo de oxidação, indicando que o comportamento de oxidação dos materiais estudados obedece à lei parabólica. Neste caso, o comportamento de oxidação é controlado por uma difusão iônica, isto é, os íons se difundem através de defeitos pontuais e a espessura da camada de óxido formado corresponde diretamente ao tempo de oxidação (MOVAHEDI-RAD et al., 2016).
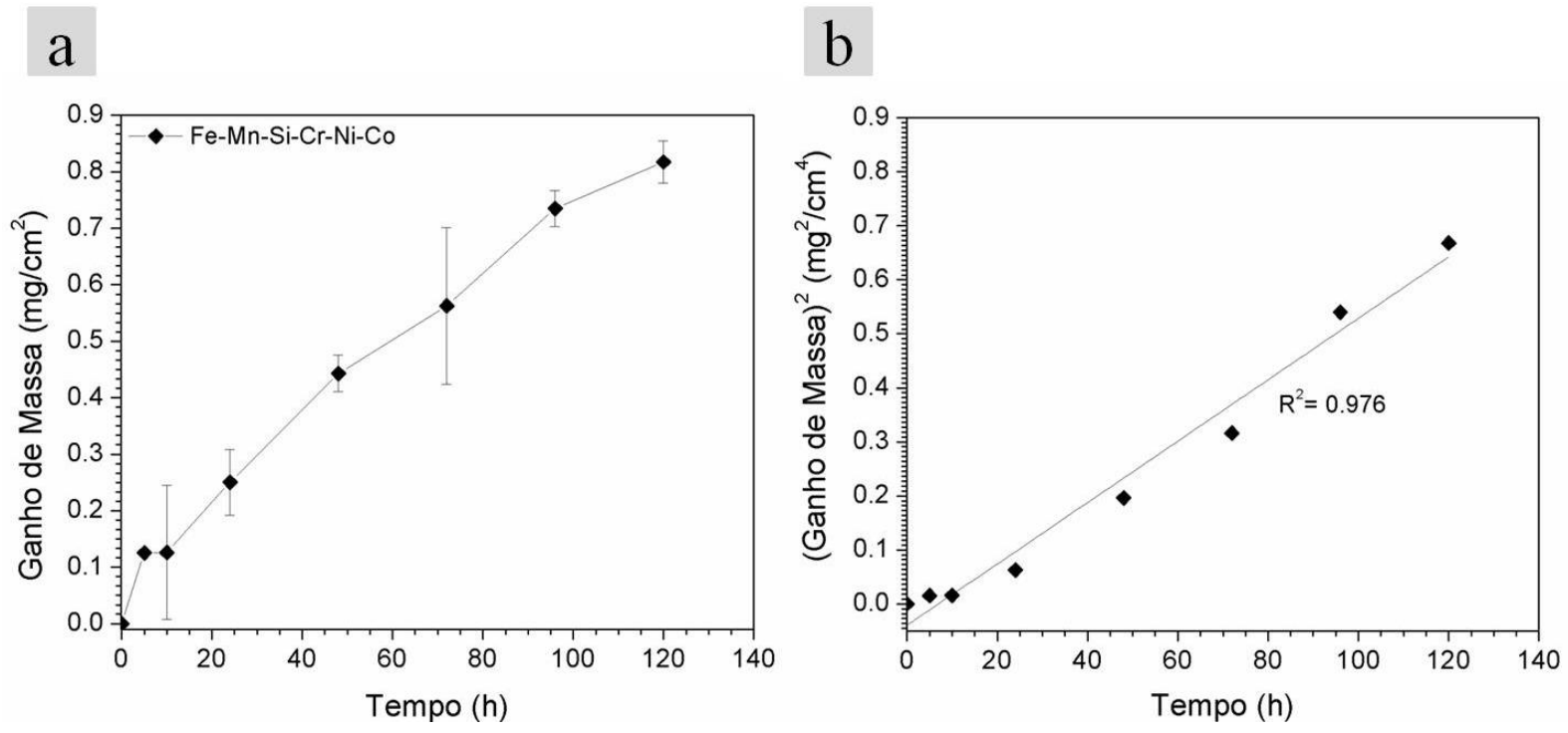

Figura 3 - Curva de ganho de massa em função do tempo de oxidação (a); Curva do ganho de massa ao quadrado em função do tempo de oxidação (b).

A Figura 4 (a) mostra imagem de MEV da seção transversal da amostra oxidada por $120 \mathrm{~h}$. É possível notar que a camada de óxido formada apresenta uma forma bastante regular (i.e., sem muitas reentrâncias e variações de espessura) chegando a aproximadamente $7 \mu \mathrm{m}$ após $120 \mathrm{~h}$ de oxidação. Pela Figura 4 (a) também ser observado a presença de algumas regiões não oxidadas, situadas abaixo da carepa de oxido, que diferem em microestrutura com relação à matriz. A Figura 4 (b), (c), (d), (e), (f), (g) e (h) apresenta o mapeamento químico elementar da seção transversal da amostra oxidada por $120 \mathrm{~h}$, pelas imagens pode ser visto que os elementos predominantes no óxido são $\mathrm{Mn}$, que se encontra uniformemente distribuído por toda a camada de óxido, e $\mathrm{Cr}$, preferencialmente distribuído na interface metal/óxido. Outro fato que também pode ser observado através do mapeamento químico elementar é que a região não oxidada situada abaixo da carepa de óxido é substancialmente empobrecida em Mn. Este resultado está diretamente relacionado com seletiva oxidação do Mn, que causa o empobrecimento deste elemento em regiões adjacentes à interface metal/óxido, causando nestas regiões uma transformação de fase de austenita para ferrita (COCCIA et al., 1997; MA et al., 2013). 

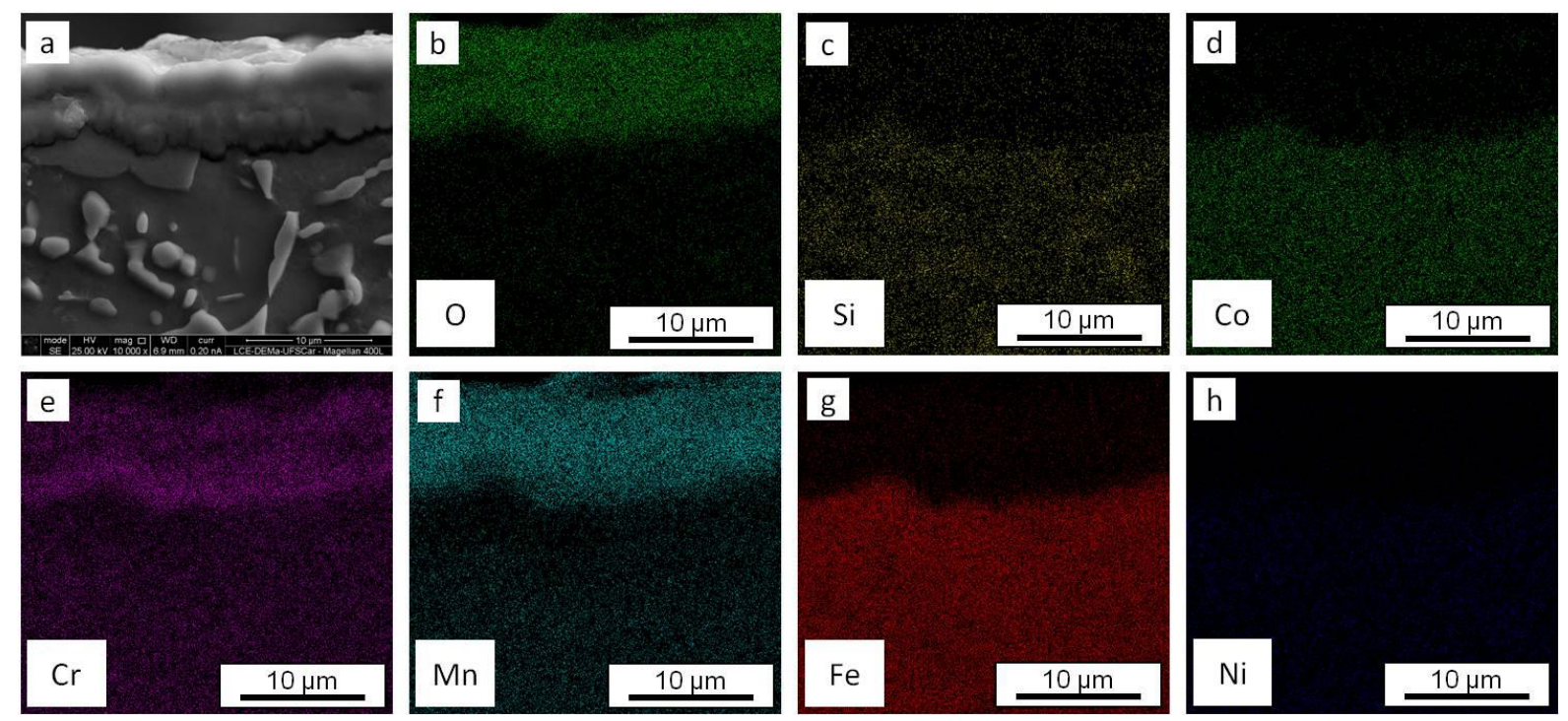

Figura 4 - Imagem de MEV da seção transversal da amostra oxidada por $120 \mathrm{~h}$ (a) e mapeamento químico elementar da seção transversal da amostra oxidada por $120 \mathrm{~h}$ (b), (c), (d), (e), (f), (g) e (h).

A Figura 5 apresenta o padrão de DRX da carepa de óxido formado após $120 \mathrm{~h}$ de exposição a $800{ }^{\circ} \mathrm{C}$. Note que a camada de óxido formada é composta de $\mathrm{Mn}_{2} \mathrm{O}_{3}, \mathrm{Mn}_{3} \mathrm{O}_{4}$ e $\mathrm{MnCr}_{2} \mathrm{O}_{4}$, também pode ser observado picos caraterísticos de ferrita, que como anteriormente mencionado são decorrentes da transformação de fase de austenita para ferrita em regiões empobrecidas em Mn.

A formação de uma camada de óxido composta essencialmente por Mn é resultado da preferencial oxidação deste elemento, além disso, as taxas de difusão do Mn através da camada de óxido são duas ordens de grandeza maior do que $\mathrm{Cr}$ para formar óxido na superfície (WILD, 1977). Deste modo, a camada de óxido de Mn cresce sobre uma camada mista oxidada de $\mathrm{Fe}, \mathrm{Cr}$, Si e $\mathrm{Mn}$, e com o prolongamento do tempo de oxidação ocorre a formação do espinélio de $\mathrm{MnCr}_{2} \mathrm{O}_{4}$ na camada mais interna do oxido, que é gerada pelas baixas pressões de oxigênio nesta região como também pela elevada solubilidade do Mn no óxido de Cr (COL, 2017).

Estes resultados corroboram com os reportados por Ma et al. (2013), que trabalhando com uma liga austenítica Fe-Mn14,29-Si5,57-Cr8,23-Ni4,96 com EMF oxidada na mesma temperatura por até $100 \mathrm{~h}$, observaram, através de análises de DRX, que a camada de óxido formada era composta essencialmente de $\mathrm{Mn}_{2} \mathrm{O}_{3}, \mathrm{Mn}_{3} \mathrm{O}_{4}$ e $\mathrm{MnCr}_{2} \mathrm{O}_{4}$. De acordo com os autores, a camada de óxido de $\mathrm{Mn}_{2} \mathrm{O}_{3}$ correspondia a camada mais externa, seguida por uma camada composta de $\mathrm{Mn}_{3} \mathrm{O}_{4}$ e uma camada mais interna de $\mathrm{Mn}$ e $\mathrm{Cr}$ que se combinam para formar o espinélio de $\mathrm{MnCr}_{2} \mathrm{O}_{4}$. 


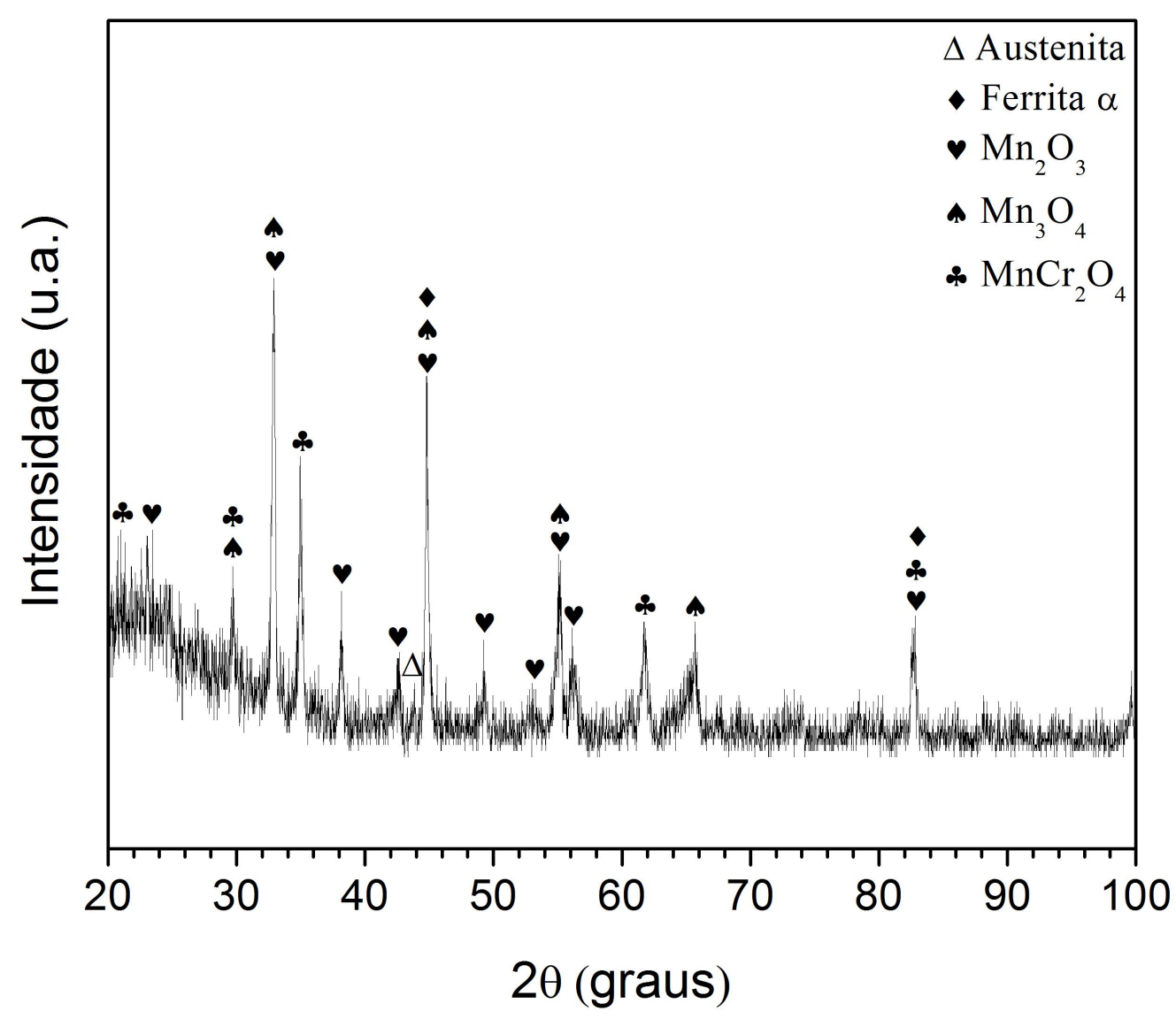

Figura 5 - Padrão de DRX da camada de óxido formada após 120 h de oxidação.

\section{CONCLUSÃO}

Com base nos resultados experimentais, podem ser tiradas as seguintes conclusões: i. O comportamento de oxidação da liga Fe-Mn-Si-Cr-Ni-Co obedece a uma lei parabólica. ii. Após 120 h de oxidação, a camada de óxido formada é composta basicamente de óxido de $\mathrm{Mn}$ do tipo $\mathrm{Mn}_{2} \mathrm{O}_{3}, \mathrm{Mn}_{3} \mathrm{O}_{4}$ e por um óxido misto de $\mathrm{MnCr}_{2} \mathrm{O}_{4}$. iii. A oxidação seletiva do $\mathrm{Mn}$ causa transformação de fase de austenita para ferrita na interface metal/óxido.

\section{AGRADECIMENTOS}

Os autores agradecem à FAPESP (Fundação de Amparo à Pesquisa do Estado de São Paulo) e CNPq (Conselho Nacional de Desenvolvimento Científico e Tecnológico - $\mathrm{N}^{\mathrm{o}}$ 460659 / 2014-6) pelo seu apoio financeiro a este trabalho. 


\section{REFERÊNCIAS}

COCCIA LECIS, G.; LENARDI, C.; SABATINI, A. The Effect of Mn depleted surface layer on the corrosion resistance of shape memory $\mathrm{FeMn}-\mathrm{Si}-\mathrm{Cr}$ alloys. Metallurgical and Materials Transactions A, Milano, Itália, v. 28A, p.1219-1222, mai. 1997.

$\mathrm{COL}$, A. et al. Oxidation of a $\mathrm{Fe}-18 \mathrm{Cr}-8 \mathrm{Ni}$ austenitic stainless steel at $850^{\circ} \mathrm{C}$ in $\mathrm{O} 2$ : Microstructure evolution during breakaway oxidation, Corrosion Science, v. 114, p. 17-27, jan. 2017.

ISSARTEL, C. et al. Influence of nitridation on the oxidation of a 304 steel at $800{ }^{\circ} \mathrm{C}$, Corrosion Science, v. 46, n. 9, p. 2191-2201, set. 2004.

KAJIWARA, S. Characteristic features of shape memory effect and related transformation behaviour in Fe-based alloys. Materials Science \& Engineering A, Tsukuba, Japan, v. 273, p. 67-88, dez. 1999.

MA, R. et al. Oxidation behavior of an austenitic stainless FeMnSiCrNi shape memory alloy. Corrosion Science, Chengdu, China, v. 66, p. 269-277, jan. 2013.

MAJI, B. C. et al. The corrosion behaviour of $\mathrm{Fe}-15 \mathrm{Mn}-7 \mathrm{Si}-9 \mathrm{Cr}-5 \mathrm{Ni}$ shape memory alloy. Corrosion Science, Mumbai, India, v. 48, p. 937-949, abr. 2006.

MOVAHEDI-RAD, A. et al. Oxidation behavior of AISI 321, AISI 316, and AISI 409 stainless steels: Kinetic, thermodynamic, and diffusion studies. Materials Research, Irã, vol. 31, n. 14, p. 2088-2096, jul. 2016.

ROVERE, C. A. D. et al. Corrosion behavior of shape memory stainless steel in acid media. Journal of Alloys and Compounds, São Carlos, SP, v. 509, p. 5376-5380, abr. 2011.

ROVERE, C. A. D. et al. Influence of alloying elements on the corrosion properties of shape memory stainless steels. Materials Chemistry and Physics, v. 133, n. 2-3, p. 668-673, abr. 2012

SILVA, R. et al. Effect of thermal aging at $475^{\circ} \mathrm{C}$ on the properties of lean duplex stainless steel 2101. Materials Characterization, São Carlos, SP, v. 114, p. 211-217, abr. 2016.

STOTT, F. H. et al. The influence of manganese on the high-temperature oxidation of ironchromium alloys, Materials Corrosion, v. 40, n. 4, p. 198-205, abr. 1989.

WILD, R. K. et al. High temperature oxidation of austenitic stainless steel in low oxygen pressure, Corrosion Science, v. 17, n. 2, p. 87-104, jan. 1977.

ZHANG, L. et al. Effect of aging on the corrosion resistance of 2101 lean duplex stainless steel. Materials Characterization, Shanghai, China, v. 60, n. 12, p. 1522-1528, ago. 2009. 